\title{
Enhancing Extractive Text Summarization with Topic-Aware Graph Neural Networks
}

\author{
Peng Cui, Le Hu, and Yuanchao Liu* \\ School of Computer Science and Technology, Harbin Institute of Technology \\ Harbin 150001, China \\ \{pcui, lhu, lyc\}@insun.hit.edu.cn
}

\begin{abstract}
Text summarization aims to compress a textual document to a short summary while keeping salient information. Extractive approaches are widely used in text summarization because of their fluency and efficiency. However, most of existing extractive models hardly capture intersentence relationships, particularly in long documents. They also often ignore the effect of topical information on capturing important contents. To address these issues, this paper proposes a graph neural network (GNN)-based extractive summarization model, enabling to capture intersentence relationships efficiently via graph-structured document representation. Moreover, our model integrates a joint neural topic model (NTM) to discover latent topics, which can provide document-level features for sentence selection. The experimental results demonstrate that our model not only substantially achieves state-of-the-art results on CNN/DM and NYT datasets but also considerably outperforms existing approaches on scientific paper datasets consisting of much longer documents, indicating its better robustness in document genres and lengths. Further discussions show that topical information can help the model preselect salient contents from an entire document, which interprets its effectiveness in long document summarization.
\end{abstract}

\section{Introduction}

Text summarization is an important task in natural language processing, which can help people rapidly acquire important information from a large sum of documents. Previous summarization approaches can be mainly classified into two categories, which are abstractive and extractive. Neural-based abstractive models usually use a seq2seq framework (Sutskever et al., 2014) to generate a word-by-word summary after encoding a full document. By contrast, extractive models directly select important sentences from the original document and then aggregate them into a summary. Abstractive models are generally more flexible but may produce disfluent or ungrammatical summary texts (Liu and Lapata, 2019b), whereas extractive models have advantages in factuality and efficiency (Cao et al., 2018).

Despite their success, modeling long-range inter-sentence relationships for summarization remains a challenge (Xu et al., 2019b). Hierarchical networks are usually applied to tackle this problem by modeling a document as a sequence of sequences (Cohan et al., 2018; Zhang et al., 2019). However, empirical observations (Liu and Lapata, 2019a) showed that the use of such a paradigm to model intersentence relationships does not provide much performance gain for summarization. Hierarchical approaches are also slow to train and tend to overfit (Xiao and Carenini, 2019). Most recently, Graph Neural Networks (GNNs) are widely explored to model cross-sentence relationships for summarization task. The critical step of this framework is to build an effective document graph. Several studies (Xu et al., 2019a; Yasunaga et al., 2017) built document graphs based on discourse analysis. However, this approach depends on external tools and may lead to other problems, such as semantically fragmented output (Liu et al., 2019). Wang and Liu (2020) built a word-sentence document graph based on word appearance, but such statistical graph-building approach hardly captures semantic-level relationships. Therefore, how to model a document as a graph for summarization effectively remains an open question.

\footnotetext{
${ }^{*}$ Corresponding author

This work is licensed under a Creative Commons Attribution 4.0 International License. License details: http: //creativecommons.org/licenses/by/4.0/.
} 
Another critical point of summarization is modeling global information, which plays a key role in sentence selection (Xiao and Carenini, 2019). Pre-trained language models can considerably boost the performance of summarization (Liu and Lapata, 2019a; Zhang et al., 2019) since they effectively capture context features. However, they are poor at modeling document-level information, particularly for long documents, because most of them are designed for sentences or a short paragraph (Xu et al., 2019b).

To tackle the abovementioned weaknesses, this paper proposes a novel graph-based extractive summarization model. First, we encode an entire document with a pre-trained BERT (Devlin et al., 2019) to learn contextual sentence representations, and discover latent topics with a joint neural topic model (NTM; Miao et al., 2017; Srivastava and Sutton, 2017). Second, we build a heterogeneous document graph consisting of sentence and topic nodes, and simultaneously update their representations with a modified graph attention network (GAT; Veličković et al., 2017). Third, the representations of sentence nodes are extracted to compute the final labels. Intuitively, our topic-sentence document graph has the following advantages: 1) During the graph propagation, sentence representations can be enriched by topical information, which can be considered as a kind of document-level feature and help our model distil important contents from an entire document. 2) Topic nodes can act as intermediary to bridge longdistance sentences; hence, our model can efficiently capture inter-sentence relationships. We evaluate our model on four standard datasets, including news articles and scientific papers. The experimental results show its effectiveness and superiority. To summarize, our contributions are threefold.

- We conduct a quantitative exploration on the effect of latent topics on document summarization and provide an intuitive understanding of how topical information help summarize documents.

- We propose a novel graph-based neural extractive summarization model, which innovatively incorporates latent topics into graph propagation via a joint neural topic model. To the best of our knowledge, we are the first to propose applying NTM to the extractive text summarization task.

- The experimental results demonstrate that our proposed model not only achieves competitive results compared with state-of-the-art extractive models on news datasets but also considerably outperforms existing approaches on scientific paper datasets consisting of much longer documents, indicating its better robustness in document genres and lengths.

\section{Related Work}

Neural Extractive Summarization Neural networks have achieved remarkable results in extractive summarization. Existing works mainly regard extractive summarization as a sequence labeling task (Nallapati et al., 2017; Zhang et al., 2018; Dong et al., 2018) or sentence ranking task (Narayan et al., 2018). Pre-trained language models have provided substantial performance gain for summarization (Liu and Lapata, 2019a; Zhang et al., 2019; Xu et al., 2019). In the current work, we further model inter-sentence relationships with a graph encoder and enrich sentence representations with topical information after a BERT encoder.

Graph-based Summarization Early works, such as TextRank (Mihalcea and Tarau, 2004) and LexRank (Erkan and Radev, 2004), built document graphs on the basis of inter-sentence similarity and extracted summary sentences in an unsupervised manner. Recently, the application of GNNs to document summarization has attracted considerable interests (Yasunaga et al., 2017; Xu et al., 2019b; Fernandes et al., 2018; Wang and Liu et al., 2020). Existing GNN-based summarization models build document graphs on the basis of only words or sentences. On the contrary, we explore the effects of high-level semantic units, i.e., latent topics.

Topic Modeling for Summarization Topic modeling is a powerful approach to learning document features. However, it has been rarely applied to document summarization. Wei et al. (2012) proposed to build a document graph consisting of words, sentences, and topic nodes and learn the graph with Markov chain. Zheng et al. (2019) proposed to summarize multiple documents by mining cross-document subtopics. Narayan et al. (2018) recommended enriching word representation with topical information. Unlike them, we discover latent topics with a neural topic model together with summarization. To the best of our knowledge, NTM had never been applied to extractive summarization task. 


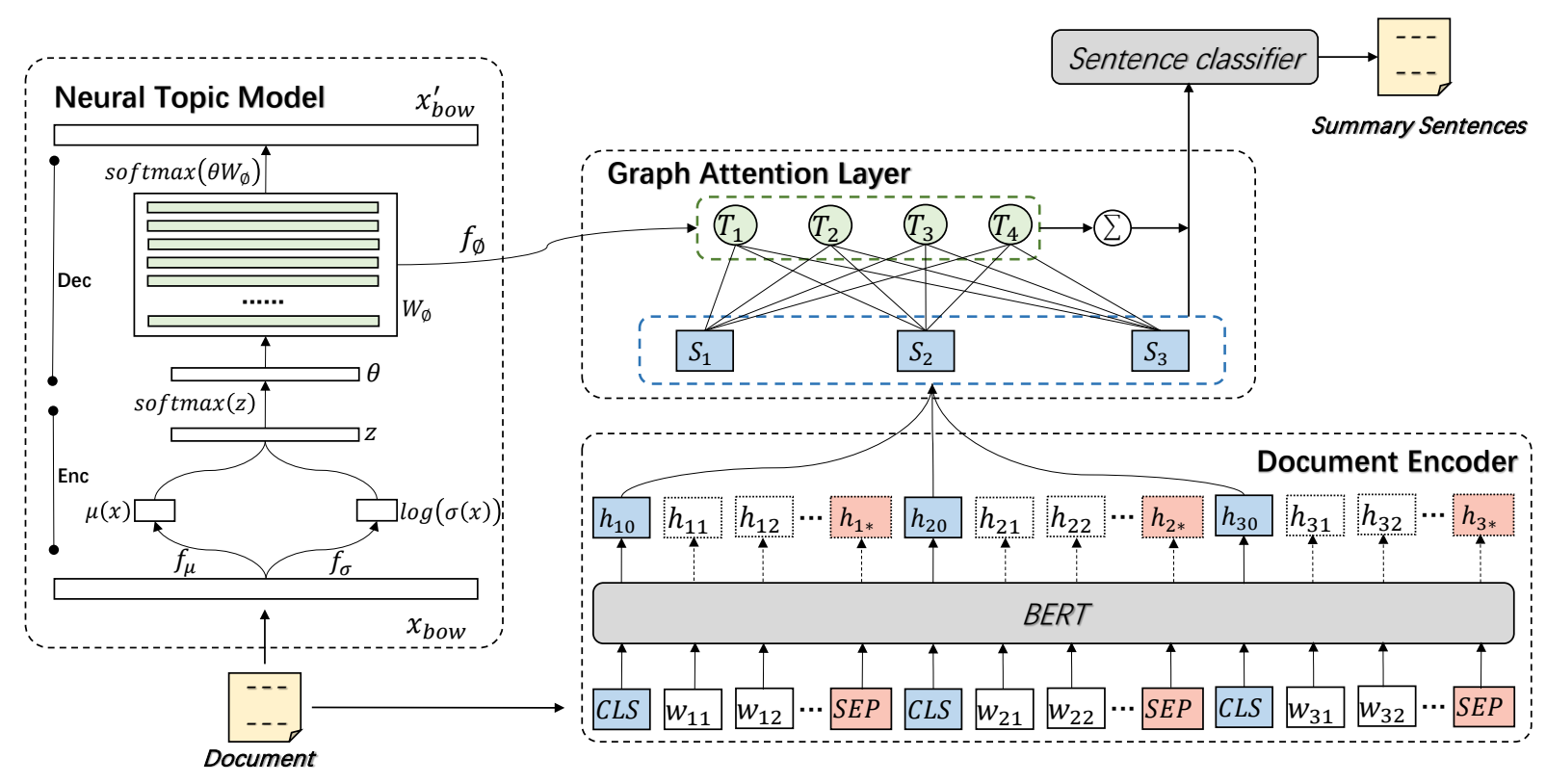

Figure 1. Overall architecture of our model (Topic-GraphSum). In the graph attention layer (top right), the square nodes denote the sentence representations output from the document encoder (bottom right), and the circular nodes denote the topic representations learned by NTM (left).

\section{Model}

This section describes our model, namely, topic-aware graph neural network for document summarization (Topic-GraphSum). Figure 1 presents the overview architecture. Given an arbitrary document $D=\left\{s_{1}, s_{2}, \ldots, s_{N}\right\}$ that consists of $N$ sentences, the objective of our model is to learn a sequence of binary labels $\left\{y_{1}, y_{2}, \ldots, y_{N}\right\}$, where $y_{i} \in\{0,1\}$ represents whether the $i$-th sentence should be included in summary. Our model generally consists of three parts, which are the 1) document encoder, 2) neural topic model, and 3) graph attention layer. Given the input document, the document encoder learns contextual representations of each sentence with a pre-trained BERT. The NTM aims to learn the document topic distribution and a group of topic representations. The graph attention layer builds a heterogeneous document graph with topics and sentences and then simultaneously update their node representations. After graph encoding, sentence representations are further combined with topics and then sent to a sentence classifier to compute the final labels. We elucidate each part below.

\subsection{Document Encoder}

BERT is a bidirectional transformer encoder pre-trained with a large corpus. Similar to previous works (Xu et al., 2019b; Liu and Lapata, 2019a), we employ a modified version of BERT to generates local context-aware hidden representations of sentences. Specifically, we insert $\langle C L S\rangle$ and $\langle S E P\rangle$ tokens at the beginning and end of each sentence, respectively. Then, we put all tokens into BERT layer and learn their hidden states.

$$
\left\{h_{1,0}, h_{1,1}, \ldots, h_{N, 0}, \ldots, h_{N, *}\right\}=\operatorname{BERT}\left(w_{1,0}, w_{1,1}, \ldots, w_{N, 0}, \ldots, w_{N, *}\right),
$$

where $w_{i, j}$ represents the $j$-th word of the $i$-th sentence. $w_{i, 0}$ and $w_{i, *}$ represent the $\langle C L S\rangle$ and $<S E P>$ tokens of the $i$-th sentence, and $h_{i, j}$ represents the hidden state of the corresponding token.

After the BERT encoding, we regard the hidden states of $\left\langle C L S>: \boldsymbol{H}_{\boldsymbol{B}}=\left\{h_{1,0}, \ldots, h_{N, 0}\right\}\right.$ as the corresponding sentence contextual representations, which will be further enriched by topic information.

\subsection{Neural Topic Model}

NTM is based on the Variational Autoencoder (VAE; Kingma and Welling, 2013) framework. It learns the latent topic via an encoding-decoding process. Let $x_{\text {bow }} \in \mathbb{R}^{|V|}$ be the bag-of-words representation 
of a given document, where $V$ is the vocabulary. In the encoder, we have $\mu=f_{\mu}(x), \log \sigma=f_{\sigma}(x)$, where $\mu$ and $\sigma$ are the prior parameters for parameterizing topic distribution in decoder networks. Functions $f_{\mu}$ and $f_{\sigma}$ are linear transformations with ReLU activation.

The decoder can be regarded as a three-step document generation process. First, we employ Gaussian softmax (Miao et al., 2017) to draw topic distribution, i.e., $z \sim \mathcal{N}\left(\mu, \sigma^{2}\right), \theta=\operatorname{softmax}(z)$, where $z$ is the latent topic variable, $\theta \in \mathbb{R}^{K}$ is the topic distribution, and $K$ is the predefined topic number. Second, we learn the probability of predicted words $p_{w} \in \mathbb{R}^{|V|}$ throughout $p_{w}=\operatorname{softmax}\left(W_{\varnothing} \theta\right)$. $W_{\varnothing} \in \mathbb{R}^{|V| \times K}$ is analogous to the topic-word distribution matrix in LDA-style topic models, and $W_{\varnothing}^{(i, j)}$ represents the relevance between the $i$-th word and $j$-th topic. Finally, we draw each word from $p_{w}$ to reconstruct input $x_{\text {bow }}$. We leave out the details and refer the readers to Miao et al. (2017).

Considering the intermediate parameters $W_{\varnothing}$ and $\theta$ have encoded topical information, we further use them to build topic representations as follows:

$$
\begin{gathered}
\boldsymbol{H}_{\boldsymbol{T}}=f_{\varnothing}\left(W_{\varnothing}^{T}\right), \\
T_{d}=\sum_{1 \leq i \leq K} \theta^{(i)} \boldsymbol{H}_{T}^{(i)},
\end{gathered}
$$

where $\boldsymbol{H}_{\boldsymbol{T}} \in \mathbb{R}^{K \times d_{t}}$ represents a group of topic representations with a predefined dimension of $d_{t}$, and $f_{\varnothing}$ is a linear transformation with ReLU activation. $T_{d} \in \mathbb{R}^{d_{t}}$ is the weighted sum of each topic representation, which can be regarded as the overall topic representation of document.

$\boldsymbol{H}_{\boldsymbol{T}}$ and $T_{d}$ are used in the graph attention layer to enrich sentence representation. Other summarization approaches (Zheng et al., 2019; Narayan et al., 2018) with topical information learn topic as a fixed feature from an external model. In comparison with them, the latent topic of our model is learned via a neural approach and can be dynamically updated with entire networks.

\subsection{Graph Attention Layer}

Graph Building Let $G=\{V, E\}$ represent an arbitrary graph, where $V$ represents the node set and $E$ represents the edge set. Formally, our undirected graph can be defined as. $V=V_{S} \cup V_{T} ; E=$ $\left\{e_{1,1}, \ldots, e_{N, K}\right\}$, where $V_{S}=\left\{s_{1}, s_{2}, \ldots, s_{N}\right\}$ stands for $N$ sentence nodes and $V_{T}=\left\{T_{1}, T_{2} \ldots, T_{K}\right\}$ stands for $K$ topic nodes. $e_{i, j}$ represents the edge between the $i$-th sentence and $j$-th topic, indicating that our document graph is bipartite.

Graph Propagation We initialize the vectors of sentence nodes and that of topic nodes with $\boldsymbol{H}^{\boldsymbol{B}}$ learned from the document encoder and $\boldsymbol{H}^{\boldsymbol{T}}$ learned from NTM (Eq. 2), respectively. Then, we update node representations with graph attention network, which can be denoted as:

$$
\begin{gathered}
z_{i, j}=\operatorname{LeaklyReLU}\left(W_{a}\left[W_{e} h_{i} ; W_{e} h_{j}\right]\right), \\
\alpha_{i, j}=\frac{\exp \left(z_{i, j}\right)}{\sum_{l \in \mathcal{N}_{i}} \exp \left(z_{i, l}\right)}, \\
u_{i}=\sum_{j \in \mathcal{N}_{i}} \tanh \left(\alpha_{i, j} W_{c} h_{j}\right), \\
h_{i}^{\prime}=\|_{m=1}^{M} \sum_{j \in \mathcal{N}_{i}} \tanh \left(\alpha_{i, j}^{m} W_{c}^{m} h_{j}\right),
\end{gathered}
$$

where $h_{i}$ is the $i$-th node representation, and $\mathcal{N}_{i}$ represents its neighbor nodes. $\| *$ represents multiheads concatenation. $W_{a}, W_{e}$, and $W_{c}$ are model trainable parameters.

The vanilla GAT is designed for homogeneous graphs. However, our document graph is heterogeneous because the sentence and topic should be considered different semantic units; hence, we need to make some adaptation. Inspired by $\mathrm{Hu}$ et al. (2019), we consider a convenient approach to project the topic and sentence representations into an implicit common space, in which we calculate the attention weight. Let $h_{i}$ be the $i$-th sentence node and $h_{j}$ be the $j$-th topic node. We modify Eq. 4 by replacing shared matrix $W_{e}$ with different projection functions, as shown as follows: 


$$
z_{i, j}=\operatorname{LeaklyReLU}\left(W_{a}\left[f_{S}\left(h_{i}\right) ; f_{T}\left(h_{j}\right)\right]\right),
$$

where $f_{S}$ and $f_{T}$ are the nonlinear transformation functions to project sentence and topic nodes to a common vector space, respectively.

The graph attention layer can build semantic relationships between sentences and topics. For example, during graph propagation, sentences can enrich their representation with topical information, which can be regarded as a global feature. Topics can capture their related sentences and distil salient contents from an entire document by their different topical relevance. Meanwhile, topic nodes can act as intermediary to help build inter-sentence relationships because they are high-level semantic units across sentences.

After graph encoding, we obtain topic-sensitive sentence representations. We concatenate them with overall topic representation $T_{d}$ (Eq. 3) to further capture their topical relevance to the document. Then, we choose a single feed-forward layer as the sentence classifier ${ }^{1}$ to predict the final labels, i.e., $\hat{y}_{i}=$ $\sigma\left(\left[h_{i}: T_{d}\right]\right)$, where $\sigma(*)$ is the sigmoid function.

\subsection{Joint Training}

We jointly train NTM and sentence classifier. For the NTM, the objective function is defined as the negative evidence lower bound, as shown as follows:

$$
\mathcal{L}_{N T M}=D_{K L}(p(z) \| q(z \mid x))-\mathbb{E}_{q(z \mid x)}[p(x \mid z)],
$$

where the first term indicates the Kullback-Leibler divergence loss, and the second term indicates the reconstruction loss. $q(z \mid x)$ and $p(x \mid z)$ represent the encoder and decoder networks, respectively.

The binary cross-entropy loss of the sentence classifier is expressed as:

$$
\mathcal{L}_{S C}=\sum_{i=1}^{n}\left(y_{i} \log \left(\hat{y}_{\mathrm{i}}\right)+\left(1-y_{i}\right) \log \left(1-\hat{y}_{i}\right)\right),
$$

The final loss of our model is the linear combination of two parts of loss with hyperparameter $\lambda$ to balance their weights, i.e.,

$$
\mathcal{L}=\mathcal{L}_{S C}+\lambda \mathcal{L}_{N T M}
$$

\section{Experimental Setup}

\subsection{Datasets}

We conduct experiments on four datasets, including two document types, which are news article and scientific paper. The summarization of news articles has been widely explored, but that of much longer scientific papers is more challenging since accurately encoding long texts for summarization is a known challenge (Vaswani et al., 2017; Frermann and Klementiev, 2019). Therefore, we conduct experiments on scientific paper datasets to verify the generalization capability of our model for long documents. The detailed statistics of four datasets is summarized in Table 1.

\begin{tabular}{c|c|ccc|cc}
\hline \multirow{2}{*}{ Datasets } & \multirow{2}{*}{ Source } & Train & \# Docs & Test & \multicolumn{2}{c}{ \# Avg. Tokens } \\
& & Doc. & Sum. \\
\hline CNN & News & 90,266 & 1,220 & 1,093 & 761 & 46 \\
Daily Mail & News & 196,961 & 12,148 & 10,397 & 653 & 55 \\
NYT & News & 96,834 & 4,000 & 3,452 & 800 & 46 \\
\hline arXiv & Scientific Paper & 203,037 & 6,436 & 6,440 & 4,938 & 220 \\
PubMed & Scientific Paper & 119,924 & 6,633 & 6,658 & 3,016 & 203 \\
\hline
\end{tabular}

Table 1: Statistics of four datasets: split size, average tokens of document and summary.

\footnotetext{
${ }^{1}$ We also tried adding more advanced classifiers (e.g., CNN and RNN) on top of GAT layer. However, the performance shows no substantial gain, indicating that our model has already learned sufficient features.
} 
CNN/DailyMail (Hermann et al., 2015) is the most widely used standard dataset for document summarization. We use standard splits and preprocess data in accordance with previous works (See et al., 2017; Liu and Lapata, 2019a; Wang and Liu, 2020).

NYT (Sandhaus, 2008) is another popular summarization dataset. It is collected from New York Times Annotated Corpus. We preprocess and divide this dataset according to Durrett et al. (2016).

arXiv and PubMed (Cohan et al., 2018) are two newly constructed datasets for long document summarization, which are collected from arXiv.org and PubMed.com, respectively. Xiao and Carenini (2019) created oracle labels for the two datasets. We use the same split as that of Cohan et al. (2018).

\subsection{Models for Comparison}

NeuSum (Zhou et al., 2018) is a neural extractive model based on seq2seq framework with attention mechanism.

BanditSum (Dong et al., 2018) regards sentence selection as a contextual bandit problem. Policy gradient methods are used to train the model.

JECS (Xu and Durrett, 2019) is a compression-based summarization model that selects sentences and compresses them by pruning a dependency tree to reduce redundancy.

BERTSUM (Liu and Lapta, 2019a) inserts multiple segmentation tokens into document to obtain each sentence representation. It is the first BERT-based extractive summarization model. We employ its framework as the basic document encoder of our model.

HiBERT (Zhang et al., 2019) modifies BERT into a hierarchical structure and design an unsupervised method to pre-train it.

DISCOBERT (Xu et al., 2019b) is a state-of-the-art BERT-based extractive model which encodes documents with BERT and then updates sentence representations with a graph encoder. DISCOBERT builds a document graph with only sentence units based on discourse analysis, whereas our model incorporates latent topics into a document graph and produce a heterogeneous bipartite graph.

\subsection{Implementation Details}

Hyperparameters For the document encoder, we use "bert-base-uncased" as our pre-trained BERT version and fine-tune it for all experiments. We also implement a non-BERT version of our model by replacing the pre-trained BERT with a Bi-GRU (Chung et al., 2014) layer and set its hidden size to 768 to compare with baseline approaches without pre-trained language models fairly. For NTM, we set topic number $K=50$. The dimension size of topic representation is set to 512 . We implement GNNs with DGL (Wang et al., 2019b), and the number of GAT layer is set to 2. We set the number of attention heads to 4 for topic nodes and 6 for sentence nodes with the same hidden size of 128 to keep the dimension size of node representations unchanged. We train our model for 500 epochs with 2 NVIDIA V100 cards, and the batch size is set to 8 . Except for the pre-trained BERT encoder, other parameters are randomly initialized and optimized using Adam (Kingma and Ba, 2014). $\lambda$ (Eq. 11) is set to 0.85 to balance the loss of topic modeling and sentence selection. All the hyperparameters are selected via grid search on the validation set with "Rouge-2" as metric.

Training Strategy We consider some empirical training strategies similar with (Cui et al., 2019) to make our model efficiently converge. Specifically, we pre-train NTM for 200 epochs with a learning rate of $1 e-3$, considering its convergence speed is much slower than that of general neural networks. In joint training, the NTM parameters are trained with a learning rate of $5 e-4$, while the learning rate of other parameters is set to $1 e-3$ because the NTM is relatively stable.

\section{Result and Analysis}

This section reports our experimental results. We evaluate our model on two criteria: 1) Whether it can achieve state-of-the-art results? 2) What benefits does the latent topic contribute to summarization? To this end, we first compare our model with state-of-the-art approaches on two widely used benchmark datasets CNN/DM and NYT. Then, we evaluate our model on two scientific paper datasets to verify whether discovering latent topics can help summarize long documents. Lastly, we present ablation and case studies for further analysis. 


\subsection{Overall Performance}

Table 2 presents the Rouge F1 results of different models on CNN/DM and NYT datasets. The first section reports the Lead-3 and Oracle; the second section reports the approaches without pre-trained language models; the third section reports BERT-based models; and the last section reports our models. From the results, we make the following observations. (1) When removing pre-trained language model, the Bi-GRU version of our model outperforms all non-BERT baseline models and obtains competitive results compared with basic BERT on both datasets. (2) Our model achieves state-of-the-art results on NYT dataset, and its performance on CNN/DM dataset is on par with DISCOBERT, which is a state-ofthe-art BERT-based extractive summarization model. It needs to mention that DISCOBERT relies on external discourse analysis for modeling long-range dependencies. Our model achieves highly competitive results without external tools, which proves its inherent superiority.

\begin{tabular}{c|c|c|c|c|c|c}
\hline \multirow{2}{*}{ Model } & \multicolumn{3}{|c|}{ CNN/DM } & \multicolumn{3}{c}{ NYT } \\
\cline { 2 - 7 } & R-1 & R-2 & R-L & R-1 & R-2 & R-L \\
\hline Lead-3 & 40.42 & 17.62 & 36.67 & 41.80 & 22.60 & 35.00 \\
Oracle & 55.61 & 32.84 & 51.88 & 64.22 & 44.57 & 57.27 \\
\hline NeuSum (Zhou et al., 2018) & 41.59 & 19.01 & 37.98 & - & - & - \\
BanditSum (Dong et al., 2018) & 41.50 & 18.70 & 37.60 & - & - & - \\
JECS (Xu and Durrett, 2019) & 41.70 & 18.50 & 37.90 & 45.50 & 25.30 & 38.20 \\
\hline BERT (Zhang et al., 2019) & 41.82 & 19.48 & 38.30 & 48.38 & 29.04 & 40.53 \\
BERTSUM (Liu and Lapata, 2019) & 43.25 & 20.24 & 39.63 & - & - & - \\
HiBERT (Zhang et al., 2019) & 42.37 & 19.95 & 38.83 & 49.06 & 29.70 & 41.23 \\
DISCOBERT (Xu et al., 2019) & 43.77 & $\mathbf{2 0 . 8 5}$ & $\mathbf{4 0 . 6 7}$ & 50.00 & 30.38 & 42.70 \\
\hline Topic-GraphSum (Bi-GRU) & 41.93 & 19.15 & 38.22 & 47.90 & 28.51 & 39.86 \\
Topic-GraphSum & $\mathbf{4 4 . 0 2}$ & 20.81 & 40.55 & $\mathbf{5 0 . 0 4}$ & $\mathbf{3 0 . 4 1}$ & $\mathbf{4 2 . 7 7}$ \\
\hline
\end{tabular}

Table 2: Rouge F1 results on the test set of CNN/DM and NYT datasets. The results of comparison models are obtained from respective papers, and - represents that corresponding result is not reported.

\subsection{Long Document Summarization}

Long documents typically cover multiple topics (Xiao and Carenini, 2019). We hypothesize that our model can capture important contents of an entire document by discovering latent topics, thus enhancing

\begin{tabular}{|c|c|c|c|c|c|c|}
\hline \multirow{2}{*}{ Model } & \multicolumn{3}{|c|}{ arXiv } & \multicolumn{3}{|c|}{ PubMed } \\
\hline & R-1 & R-2 & R-L & R-1 & R-2 & R-L \\
\hline SumBasic $^{*}$ & 29.47 & 6.95 & 26.30 & 37.15 & 11.36 & 33.43 \\
\hline LexRank $^{*}$ & 33.85 & 10.73 & 28.99 & 39.19 & 13.89 & 34.59 \\
\hline LSA $^{*}$ & 29.91 & 7.42 & 25.67 & 33.89 & 9.93 & 29.70 \\
\hline Oracle $^{+}$ & 53.88 & 23.05 & 34.90 & 55.05 & 27.48 & 38.66 \\
\hline SummaRuNNer $^{+}$ & 42.91 & 16.65 & 28.53 & 43.89 & 18.78 & 30.36 \\
\hline Seq2seq-attentive ${ }^{+}$ & 43.58 & 17.37 & 29.30 & 44.81 & 19.74 & 31.48 \\
\hline Seq2seq-cancat ${ }^{+}$ & 43.62 & 17.36 & 29.14 & 44.85 & 19.70 & 31.43 \\
\hline Cheng \& Lapata (2019) & 42.24 & 15.97 & 27.88 & 43.89 & 18.53 & 30.17 \\
\hline Attn-Seq2Seq ${ }^{*}$ & 29.30 & 6.00 & 25.56 & 31.55 & 8.52 & 27.38 \\
\hline Pntr-Gen-Seq2Seq ${ }^{*}$ & 32.06 & 9.04 & 25.16 & 35.86 & 10.22 & 29.69 \\
\hline Discourse-aware $^{*}$ & 35.80 & 11.05 & 31.80 & 38.93 & 15.37 & 35.21 \\
\hline Topic-GraphSum (Bi-GRU) & 44.71 & 18.84 & 32.58 & 46.13 & 20.91 & 33.27 \\
\hline Topic-GraphSum & 46.05 & 19.97 & 33.61 & 48.85 & 21.76 & 35.19 \\
\hline
\end{tabular}

Table 3: Rouge F1 results on the test set of arXiv and PubMed datasets. Results with * are token from Cohan et al. (2018), and results with + are token from Xiao and Carenini (2019). 
the summarization performance. To verify this hypothesis, we conduct additional experiments on longform documents. Table 3 presents the results of our model and state-of-the-art public summarization systems on arXiv and PubMed datasets. The first section includes traditional approaches and Oracle; the second and third sections include abstractive and extractive models, respectively. From Table 3, our model substantially outperforms baseline models by a large margin without pre-trained BERT, and the gaps further increase when combined with BERT. We note that discourse-aware model (Cohan et al., 2018) slightly outperforms our model on R-L of PubMed dataset; a possible reason is that it explicitly leverages the section information (e.g., introduction and conclusion) of papers, which may be strong clues in selecting summary sentences. Our model achieves state-of-the-art performance on scientific paper datasets without additional features, indicating that discovering latent topics can indeed help summarize long document, consistent with aforementioned analysis.

\subsection{Ablation Study}

To analyze the relative contributions of different modules in summarizing documents, we compare our full model with three ablated variants: 1) w/o NTM, which removes the NTM module, builds a document graph with fully connected sentence nodes, and can be regarded as performing self-attention calculation on the top of BERT; 2) w/o GAT, which removes the graph attention layer, directly concatenates each of sentence representation with overall topic vector $T_{d}$ (Eq. 3), and sends them to the sentence classifier; and 3) LDA Version, which replaces NTM with standard LDA and randomly initializes each topic representation.

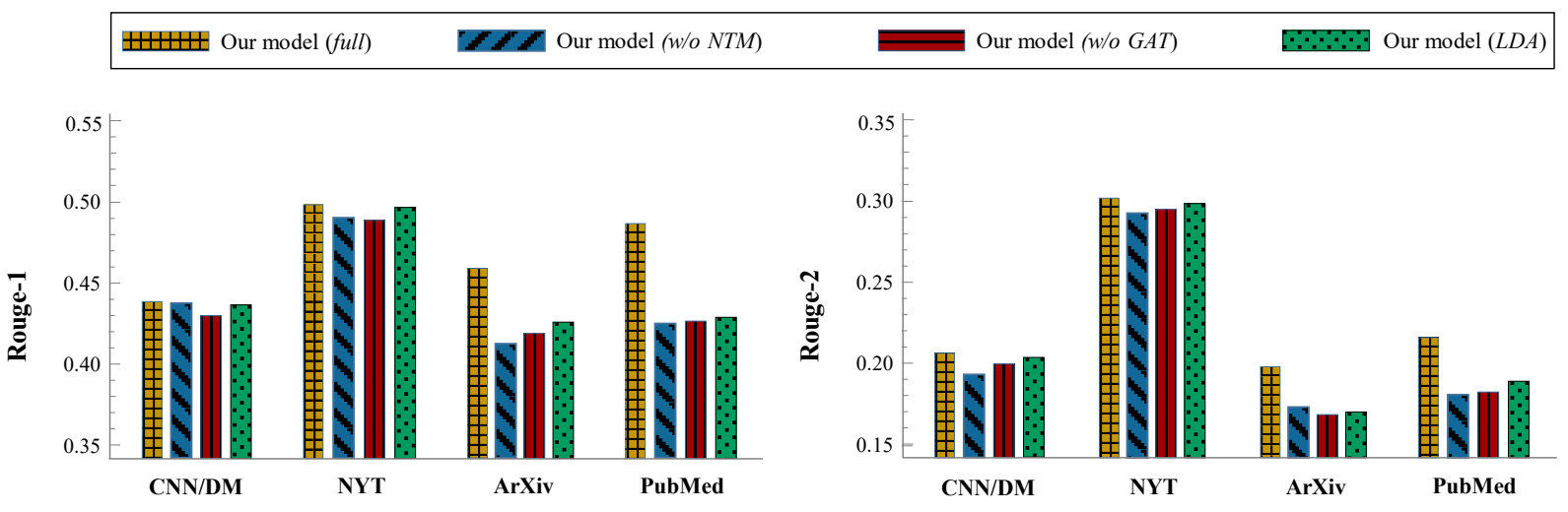

Figure 2. Rouge-1 and -2 results of our full model and three ablated variants on four datasets.

Figure 2 shows the results of different variants on four datasets, from which we can make the following observations. 1) Our full model outperforms all variants on four datasets, which proves that each module is necessary and combining them can help our model achieve the best performance. 2) When NTM module is removed or using LDA instead, the performance on arXiv and PubMed datasets declines dramatically, whereas on CNN/DM and NYT datasets, the results are still competitive with our full model. A possible reason lies in that news documents are relatively short, which leads to the data sparsity problem and thus reduces the effect of topic models. 3) Similarly, when GAT is removed, the performance of scientific paper datasets has decreased more significantly than that of news datasets. This phenomenon indicates that inter-sentence relationships are especially important for summarizing long documents. 4) The LDA topic model can also boost the performance, but the gain of LDA is much fewer than that of NTM for long documents; a possible reason is that LDA and neural networks are inevitably disconnected, whereas NTM can be jointly optimized with the document encoder and graph networks, which can mutually improve each module (Wang et al., 2019).

\subsection{Analysis of Latent Topics}

In this subsection, we conduct experiments to better understand how latent topics help summarize documents. To this end, we define the topical weight of a sentence as the weighted summation of attention score between each topic and the sentence, i.e., 


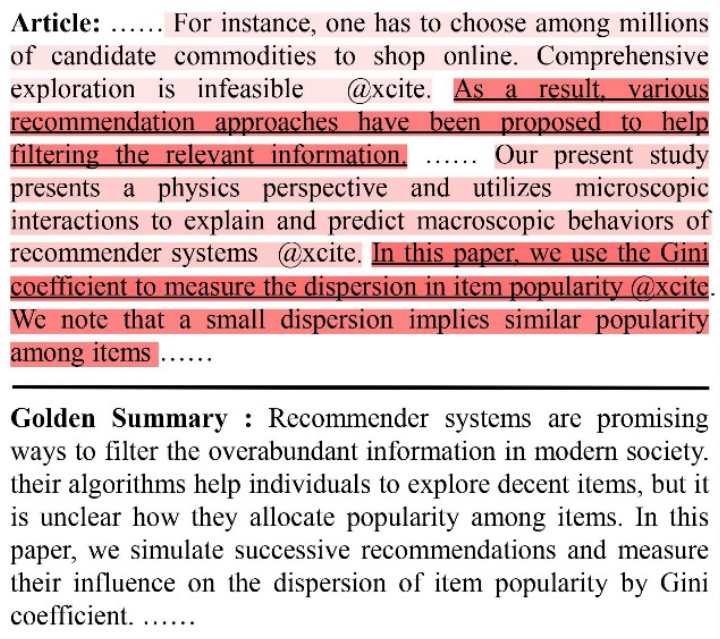

Golden Summary : Recommender systems are promising ways to filter the overabundant information in modern society. their algorithms help individuals to explore decent items, but it is unclear how they allocate popularity among items. In this paper, we simulate successive recommendations and measure their influence on the dispersion of item popularity by Gini coefficient. ......

Article: ...... One in a million: New York alone is believed to be
home to more than one million pigeons. I've also had the cops
called on me on three separate occasions while I was birding on
my own block. Feral pigeons find the ledges of buildings to be a
substitute for sea cliffs, have become adapted to urban life, and
are abundant in towns and cities throughout much of the
world. ...... This was huge because it enabled me to start
catching the birds in flight. I like when the pigeons dip below the
horizon, this way you have the nice contrast of the crisp bird in
focus with the colorful, dreamy bokeh [blur] of the backdrop.
Pigcons are exceptional flicrs, they are constantly on the look out
for predators and must cooperate with cach other to stay on top of
these threats. ......
Golden Summary : New York alone is believed to be home to
more than one million pigeons. Feral pigeons find the ledges of
buildings to be a substitute for sea cliffs. Pigeons are exceptional
fliers, constantly on the look out for predators. They must
cooperate with each other to avoid predators.

Figure 3. Visualized results of sentence topical weight. The degree of highlighting represents the overall relevance of the sentence and all topics. Underlined sentences are model-selected summary. The left document is from PubMed dataset, and the right document is from CNN/DM dataset.

$$
T W_{i}=\sum_{j=1}^{K} \theta^{(j)} \alpha_{j, i}
$$

where $T W_{i}$ represents the topical weight of the $i$-th sentence. $\theta$ is the topic distribution of the document learned by NTM described in Section 3.2, and $\theta^{(j)}$ represents the weight of $j$-th topic in document. $\alpha_{j, i}$ (Eq. 5) is the attention score from the $j$-th topic node to the $i$-th sentence node.

Figure 3 shows two examples of visualized sentence topical weights. The ground-truth summary sentences have relatively high topical weights, and the final selected sentences highly overlap with these topical sentences. From such observation, we can have an intuitive understanding of how our model works. First, our model learns sentence representations and discovers latent topics, individually. Second, the graph attention layer builds semantic relationships between sentences and topics and then roughly selects important contents on the basis of topical information. Finally, our model accurately selects summary sentences by integrating all features, such as the topical relevance to the document, context information, and inter-sentence relationships. This process may explain why our model is effective for long documents. Latent topics can help our model preselect salient texts; thus, further selection can mainly focus on these fragments rather than entire document.

\section{Conclusion and Future Work}

In this paper, we systematically explore the effects of latent topics for document summarization, and propose a novel graph-based extractive summarization model, which allows joint learning of latent topics and leverages them to enrich sentence representations via a heterogeneous graph neural network. The experimental results on four well-studied datasets demonstrate that our model not only achieves results on par with state-of-the-art summarization models on news article datasets but also significantly outperforms existing approaches on scientific paper datasets, indicating its strong robustness in various document genres and lengths. Further explorations on incorporating more types of semantic units (e.g. keywords and entities) into document graph for enhancing the performance of summarization will be addressed in our future work.

\section{Acknowledgements}

This work is supported by grant from the National Natural Science Foundation of China (No. 61672192). We thank anonymous reviewers for their helpful comments on various aspects of this work. 


\section{Reference}

Alfred V. Aho and Jeffrey D. Ullman. 1972. The Theory of Parsing, Translation and Compiling, volume 1. Prentice-Hall, Englewood Cliffs, NJ.

Benjamin Borschinger and Mark Johnson. 2011. A particle filter algorithm for Bayesian word segmentation. In Proceedings of the Australasian Language Technology Association Workshop 2011, pages 10-18, Canberra, Australia.

Peng Cui, Yuanchao Liu, Bingquan Liu. 2019. A neural topic model based on variational auto-encoder for aspect extraction from opinion texts. In Natural Language Processing and Chinese Computing. NLPCC 2019. Lecture Notes in Computer Science, vol 11838.

Arman Cohan, Franck Dernoncourt, Doo Soon Kim, Trung Bui, Seokhwan Kim, Walter Chang, and Nazli Goharian. 2018. A discourse-aware attention model for abstractive summarization of long documents. In Proceedings of NAACL-HLT 2018, pages 615-621 New Orleans, Louisiana. Association for Computational Linguistics.

Ashok K. Chandra, Dexter C. Kozen, and Larry J. Stockmeyer. 1981. Alternation. Journal of the Association for Computing Machinery, 28(1):114-133.

Ziqiang Cao, Furu Wei, Wenjie Li, and Sujian Li. 2018. Faithful to the Original: Fact Aware Neural Abstractive Summarization. In AAAI Conference on Artificial Intelligence.

Junyoung Chung, Caglar Gulcehre, Kyunghyun Cho, and Yoshua Bengio. 2014. Empirical Evaluation of Gated Recurrent Neural Networks on Sequence Modeling. In NIPS.

Greg Durrett, Taylor Berg-Kirkpatrick, and Dan Klein. 2016. Learning-based single-document summarization with compression and anaphoricity constraints. arXiv preprint arXiv:1603.08887.

Jacob Devlin, Ming-Wei Chang, Kenton Lee, and Kristina Toutanova. 2019. BERT: Pre-training of deep bidirectional transformers for language understanding. In Proceedings of the 2019 Conference of the North American Chapter of the Association for Computational Linguistics: Human Language Technologies, Volume 1 (Long and Short Papers), pages 4171-4186, Minneapolis, Minnesota.

Yue Dong, Yikang Shen, Eric Crawford, Herke van Hoof, and Jackie Chi Kit Cheung. 2018. BanditSum: Extractive Summarization as a Contextual Bandit. In Proceedings of the 2018 Conference on Empirical Methods in Natural Language Processing, pages 3739-3748. Association for Computational Linguistics.

Gunes Erkan and Dragomir R Radev. 2004. Lexrank: “ Graph-based lexical centrality as salience in text summarization. Journal of artificial intelligence research, 22:457-479.

Patrick Fernandes, Miltiadis Allamanis, and Marc Brockschmidt. Structured neural summarization. 2018. arXiv preprint arXiv:1811.01824.

Lea Frermann and Alexandre Klementiev. 2019. Inducing document structure for aspect-based summarization. In Proceedings of the 57th Annual Meeting of the Association for Computational Linguistics, pages 6263-6273 Florence, Italy. Association for Computational Linguistics.

Dan Gusfield. 1997. Algorithms on Strings, Trees and Sequences. Cambridge University Press, Cambridge, UK.

Karl Moritz Hermann, Tomas Kocisky, Edward Grefenstette, Lasse Espeholt, Will Kay, Mustafa Suleyman, and Phil Blunsom. 2015. Teaching Machines to Read and Comprehend. In C. Cortes, N. D. Lawrence, D. D. Lee, M. Sugiyama, and R. Garnett, editors, Advances in Neural Information Processing Systems 28, pages 16931701. Curran Associates, Inc.

Linmei Hu, Tianchi Yang, Chuan Shi, Houye Ji and Xiaoli Li .2019. Heterogeneous graph attention networks for semi-supervised short text classification. In Proceedings of the 2019 Conference on Empirical Methods in Natural Language Processing and the 9th International Joint Conference on Natural Language Processing, pages 4821-4830, Hong Kong, China. Association for Computational Linguistics.

Diederik Kingma and Jimmy Ba. 2014. Adam: A method for stochastic optimization. In Proceedings of the 2nd International Conference on Learning Representations.

Diederik P Kingma and Max Welling. 2013. Autoencoding variational bayes. arXiv preprint arXiv:1312.6114.

Zhengyuan Liu, Nancy F. Chen. 2019. Exploiting discourse-level segmentation for extractive summarization. In Proceedings of the 2nd Workshop on New Frontiers in Summarization, pages 116-121 Hong Kong, China. Association for Computational Linguistics. 
Yang Liu and Mirella Lapata. 2019a. Text summarization with pretrained encoders. In Proceedings of the 2019 Conference on Empirical Methods in Natural Language Processing and the 9th International Joint Conference on Natural Language Processing (EMNLP-IJCNLP), pages 3728-3738, Hong Kong, China. Association for Computational Linguistics.

Yang Liu and Mirella Lapata. 2019b. Hierarchical transformers for multi-document summarization. In Proceedings of the 57th Annual Meeting of the Association for Computational Linguistics, pages 5070-5081 Florence, Italy. Association for Computational Linguistics

Yishu Miao, Edward Grefenstette, and Phil Blunsom. 2017. Discovering discrete latent topics with neural variational inference. In Proceedings of the 34th International Conference on Machine Learning, ICML 2017, Sydney, NSW, Australia, pages 2410-2419.

Rada Mihalcea and Paul Tarau. 2004. Textrank: Bringing order into text. In Proceedings of the 2004 conference on empirical methods in natural language processing, pages 404-411.

Shashi Narayan, Shay B Cohen, Mirella Lapata. 2018. Don't Give Me the Details, Just the Summary! Topic-aware convolutional neural networks for extreme summarization. In Proceedings of the 2018 Conference on Empirical Methods in Natural Language Processing, pages 1797-1807 Brussels, Belgium. Association for Computational Linguistics.

Ramesh Nallapati, Feifei Zhai, and Bowen Zhou. 2017. SummaRuNNer: A Recurrent Neural Network Based Sequence Model for Extractive Summarization of Documents. In AAAI Conference on Artificial Intelligence.

Mohammad Sadegh Rasooli and Joel R. Tetreault. 2015. Yara parser: A fast and accurate dependency parser. Computing Research Repository, arXiv preprint arXiv:1503.06733. Version 2.

Abigail See, Peter J. Liu, and Christopher D. Manning. 2017. Get to the point: Summarization with pointergenerator networks. In Proceedings of the 55th Annual Meeting of the Association for Computational Linguistics (Volume 1: Long Papers), pages 1073-1083, Vancouver, Canada

Akash Srivastava and Charles Sutton. 2017. Autoencoding variational Inference for topic models. arXiv preprint arXiv: 1703.01488 .

Ilya Sutskever, Oriol Vinyals, and Quoc V Le. 2014. Sequence to sequence learning with neural networks. In Advances in neural information processing systems. pages 3104-3112.

Evan Sandhaus. 2008. The New York Times Annotated Corpus. Linguistic Data Consortium, Philadelphia, 6(12).

Petar Veličković, Guillem Cucurull, Arantxa Casanova, Adriana Romero, Pietro Lio, and Yoshua Bengio. 2017. Graph attention networks. arXiv preprint arXiv:1710.10903.

Ashish Vaswani, Noam Shazeer, Niki Parmar, Jakob Uszkoreit, Llion Jones, Aidan N Gomez, Ł ukasz Kaiser, and Illia Polosukhin. 2017. Attention is all you need. In I. Guyon, U. V. Luxburg, S. Bengio, H. Wallach, R. Fergus, S. Vishwanathan, and R. Garnett, editors, Advances in Neural Information Processing Systems 30, pages 59986008. Curran Associates, Inc.

Yue Wang, Jing Li, Hou Pong Chan, Irwin King, Michael R. Lyu and Shuming Shi. 2019a. Topic-aware neural keyphrase generation for social media language. In Proceedings of the 57th Annual Meeting of the Association for Computational Linguistics, pages 2516-2526 Florence, Italy. Association for Computational Linguistics.

Danqing Wang, Pengfei Liu, Yining Zheng, Xipeng Qiu and Xuanjing Huang. 2020. Heterogeneous Graph Neural Networks for Extractive Document Summarization. arXiv preprint arXiv:2004.12393.

Minjie Wang, Lingfan Yu, Da Zheng, Quan Gan, Yu Gai, Zihao Ye, Mufei Li, Jinjing Zhou, Qi Huang, Chao Ma, Ziyue Huang, Qipeng Guo, Hao Zhang, Haibin Lin, Junbo Zhao, Jinyang Li, Alexander J Smola, and Zheng Zhang. 2019b. Deep graph library: Towards efficient and scalable deep learning on graphs. ICLR Workshop on Representation Learning on Graphs and Manifolds.

Yang Wei. 2012. Document summarization method based on heterogeneous graph. In 2012 9th International Conference on Fuzzy Systems and Knowledge Discovery, pages 1285-1289. IEEE.

Wen Xiao and Giuseppe Carenini. 2019. Extractive summarization of long documents by combining global and local context. In Proceedings of the 2019 Conference on Empirical Methods in Natural Language Processing and the 9th International Joint Conference on Natural Language Processing, pages 3011-3021, Hong Kong, China. Association for Computational Linguistics.

Jiacheng Xu and Greg Durrett. 2019a. Neural extractive text summarization with syntactic compression. arXiv preprint arXiv: 1902.00863 
Jiacheng Xu, Zhe Gan, Yu Cheng, and Jingjing Liu. 2019b. Discourse-aware neural extractive model for text summarization. arXiv preprint arXiv:1910.14142.

Michihiro Yasunaga, Rui Zhang, Kshitijh Meelu, Ayush Pareek, Krishnan Srinivasan, and Dragomir Radev. 2017. Graph-based neural multi-document summarization. In Proceedings of the 21st Conference on Computational Natural Language Learning (CoNLL 2017), pages 452-462, Vancouver, Canada. Association for Computational Linguistics.

Xingxing Zhang, Mirella Lapata, Furu Wei, and Ming Zhou. 2018. Neural Latent Extractive Document Summarization. In Proceedings of the 2018 Conference on Empirical Methods in Natural Language Processing, pages 779-784. Association for Computational Linguistics.

Xin Zheng, Aixin Sun, Jing Li ,and Karthik Muthuswamy. 2019. Subtopic-driven multi-document summarization. In Proceedings of the 2019 Conference on Empirical Methods in Natural Language Processing and the 9th International Joint Conference on Natural Language Processing, pages 3153-3162, Hong Kong, China. Association for Computational Linguistics.

Xingxing Zhang, Furu Wei, and Ming Zhou. 2019. HIBERT: Document level pre-training of hierarchical bidirectional transformers for document summarization. In Proceedings of the 57th Annual Meeting of the Association for Computational Linguistics, pages 5059-5069, Florence, Italy. Association for Computational Linguistics.

Qingyu Zhou, Nan Yang, Furu Wei, Shaohan Huang, Ming Zhou, and Tiejun Zhao. 2018. Neural Document Summarization by Jointly Learning to Score and Select Sentences. In Proceedings of the 56th Annual Meeting of the Association for Computational Linguistics (Volume 1: Long Papers), pages 654- 663. Association for Computational Linguistics. 